\title{
Challenges and Advances in Genome Editing Technologies in Streptomyces
}

\author{
Yawei Zhao ${ }^{1,2}$, Guoquan $\mathrm{Li}^{3}$, Yunliang Chen ${ }^{4, *}$ and Yinhua $\mathrm{Lu}{ }^{1, *}$ \\ 1 College of Life Sciences, Shanghai Normal University, Shanghai 200234, China; zhaoyawei@sibs.ac.cn \\ 2 College of Bioengineering, Henan University of Technology, Zhengzhou 450001, China \\ 3 School of Food and Biological Engineering, Jiangsu University, Zhenjiang 212013, China; \\ 13775556788@139.com \\ 4 School of Agricultural Equipment Engineering, Zhenjiang, Jiangsu University Zhenjiang 212013, China \\ * $\quad$ Correspondence: ylchen@ujs.edu.cn (Y.C.); yhlu@shnu.edu.cn (Y.L.); Tel.: +86-21-64322208 (Y.L.)
}

Received: 25 March 2020; Accepted: 4 May 2020; Published: 8 May 2020

check for updates

\begin{abstract}
The genome of Streptomyces encodes a high number of natural product (NP) biosynthetic gene clusters (BGCs). Most of these BGCs are not expressed or are poorly expressed (commonly called silent BGCs) under traditional laboratory experimental conditions. These NP BGCs represent an unexplored rich reservoir of natural compounds, which can be used to discover novel chemical compounds. To activate silent BGCs for NP discovery, two main strategies, including the induction of BGCs expression in native hosts and heterologous expression of BGCs in surrogate Streptomyces hosts, have been adopted, which normally requires genetic manipulation. So far, various genome editing technologies have been developed, which has markedly facilitated the activation of BGCs and NP overproduction in their native hosts, as well as in heterologous Streptomyces hosts. In this review, we summarize the challenges and recent advances in genome editing tools for Streptomyces genetic manipulation with a focus on editing tools based on clustered regularly interspaced short palindrome repeat (CRISPR)/CRISPR-associated protein (Cas) systems. Additionally, we discuss the future research focus, especially the development of endogenous CRISPR/Cas-based genome editing technologies in Streptomyces.
\end{abstract}

Keywords: genome editing; CRISPR/Cas; Streptomyces; microbial natural products

\section{Introduction}

Streptomyces are Gram-positive bacteria that can produce high amounts of secondary metabolites, such as antibiotics (e.g., pristinamycin [1] and daptomycin [2]), immunosuppressants (e.g., rapamycin [3] and FK506 [4]), insecticides (e.g., avermectin [5] and milbemycin [6]) and anti-tumor drugs (e.g., daunorubicin [7] and bleomycin [8]), which are widely used in agriculture and veterinary/human medicine. In the last few decades, the traditional strategy for the discovery of novel natural products (NPs) with medicinal properties has not been effective, which is mainly due to the repeated rediscovery of known chemical compounds [9]. The bioinformatic analysis of the sequenced Streptomyces genomes has revealed the presence of a large number of NP biosynthetic gene clusters (BGCs). In each genome, there are approximately 20-50 NP BGCs, which are much more than known NPs isolated previously [10]. These NP BGCs are an unexplored rich reservoir of natural compounds, which can be used for the discovery of novel chemical compounds. However, the majority of these BGCs are not expressed or are poorly expressed (commonly called silent BGCs) in streptomycetes under traditional laboratory culture conditions [11]. Recently, various strategies have been developed to activate these silent BGCs and trigger NP overproduction, which has contributed to scientific research on the identification of potential chemical compound resources in streptomycetes [12]. Generally, these strategies can be 
broadly classified into the following two main categories: (i) the induction of BGCs expression in native Streptomyces hosts that is genetically tractable, which usually involves genetic manipulations of the host genome, such as knocking out the competitive pathways [13] and negative regulatory genes [14], replacing the native promoters with strong promoters [15], and overexpression of positive regulatory genes [16]; (ii) cloning and/or refactoring NP BGCs, followed by transfer into surrogate Streptomyces hosts for heterologous expression, which is especially useful for the activation of silent BGCs from genetically intractable streptomycetes [17]. The successful heterologous expression of these NP BGCs is always followed by strain improvement to produce high titers of target compounds. To achieve the activation of silent BGCs in either native or heterologous Streptomyces hosts, it is critical to develop highly efficient genome editing technologies.

Traditional genetic manipulation strategies for streptomycetes, including DNA deletion, disruption, and replacement, employ suicide plasmids or plasmids with temperature-sensitive replication origin (e.g., pKC1139), which require the selection and screening of single- and double-crossover recombination events, respectively [18]. The traditional strategy, which is a time-consuming process, has low efficiency for genetic engineering. Additionally, the double-crossover mutants are rarely obtained in streptomycetes, which exhibit weak DNA homologous recombination. To address these limitations, various genome editing technologies have been developed (Figure 1), especially the clustered regularly interspaced short palindromic repeat (CRISPR)/CRISPR-associated protein (Cas)-based tools, which have markedly improved the genetic manipulation of streptomycetes and accelerated NP discovery, strain improvement, and functional genome research [19]. In this review, we summarize the challenges and recent advances in key genome engineering technologies for Streptomyces with a special focus on the development of novel technologies based on CRISPR/Cas systems. We also discuss the future research focus, especially the development of genome editing tools based on endogenous CRISPR/Cas systems widely distributed in Streptomyces.

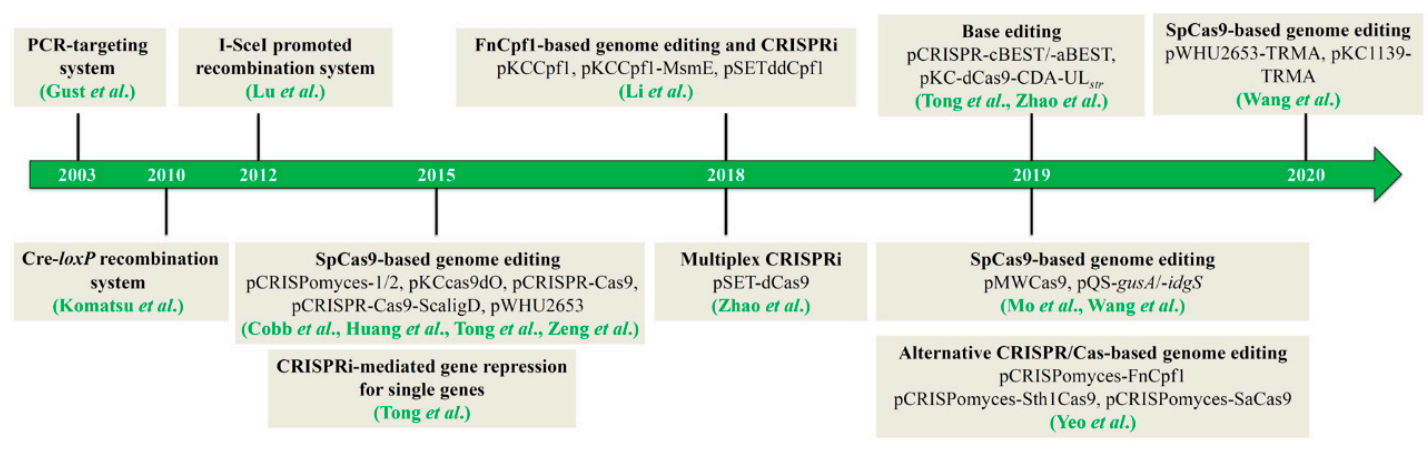

Figure 1. The development course of genome editing technologies in Streptomyces.

\section{PCR-Targeting System}

The PCR-targeting system was first developed for gene knockout in Escherichia coli, which is based on the high-efficiency recombination between the target region of the E. coli genome and a PCR-amplified selectable marker flanked at both ends by 40-50 bp homologous extensions [20]. The recombination events are mediated by the $\lambda$ recombination system $(\lambda$ Red), which includes the Red $\alpha$, $\beta$, and $\gamma$ proteins from the $\lambda$ phage [21]. Gust et al. adapted this system for nonpolar and in-frame deletion of genes or gene clusters in S. coelicolor [22]. The PCR-targeting system-assisted genetic engineering of Streptomyces involves four steps: (i) a cosmid library of the Streptomyces strain needs to be constructed; (ii) the target gene within the cosmid is knocked out by the PCR-targeting system in E. coli. To facilitate the removal of the selectable marker (e.g., the antibiotic-resistant gene), the antibiotic-resistance cassette is designed to be flanked by the FRT or loxP sites; (iii) the mutant cosmid with deletion of the target gene is introduced into Streptomyces to screen for mutant strains with double-crossover recombination events; (iv) the antibiotic-resistance cassette is removed by inducing the expression of the tyrosine recombinase FLP (FLP-FRT) or Cre (Cre-loxP). The PCR-targeting system is very efficient for the deletion of genes or gene clusters in several Streptomyces 
strains. However, the PCR-targeting system has the following three limitations, which have limited its wide application: (i) a scar (FRT or loxP site) is left on the genome of the mutant strain; (ii) a cosmid library of Streptomyces must be constructed in advance; (iii) the procedure involves four steps, which are cumbersome and time-consuming.

\section{Cre-loxP Recombination System}

The Cre-loxP recombination system can be used in combination with the PCR-targeting system for genetic engineering in Streptomyces as above. Additionally, it can be independently used to knock out large fragments of DNA in Streptomyces [23]. This system involves the following two main steps: (i) two loxP sites in the same orientation are introduced into the genome to flank the DNA fragment to be deleted by two single-crossover events; (ii) the expression of the Cre recombinase is induced to delete the DNA fragment between the two loxP sites. This method is very effective for knocking out large fragments of DNA and can be used to knock out a DNA fragment with a size larger than $1.4 \mathrm{Mb}$ in the industrial strain, Streptomyces avermitilis [23]. Similar to the PCR-targeting system, this system also generates a scar (a loxP sequence) on the genome and the genetic engineering procedure is time-consuming.

\section{I-SceI Meganuclease-Promoted Recombination System}

Conventional gene knockout methods often use the temperature-sensitive pKC1139 plasmid or segregationally unstable pJTU1278 plasmid to screen for single- and double-crossover events [24,25]. The single-crossover mutants can be easily screened based on antibiotic resistance. However, double-crossover events are often difficult to be obtained, especially in strains exhibiting weak homologous recombination. I-SceI meganuclease can recognize an 18-bp unique sequence and cause DNA double-strand breaks (DSBs), which promote double-crossover recombination events [26,27]. Lu et al. synthesized the codon-optimized I-SceI gene and developed an I-SceI-assisted genome editing technology in S. coelicolor [28]. Two BGCs that biosynthesize actinorhodin and undecylprodigiosin were successfully deleted by this technology [28,29]. Compared to the traditional gene deletion method, I-SceI cleavage markedly improves the efficiency of double-crossover events.

\section{CRISPR/Cas-Based Genome Editing}

The CRISPR/Cas system, which serves as an adaptive immune system against invading mobile genetic elements (e.g., phages and plasmids), is widely distributed in archaea and bacteria [30]. CRISPR/Cas system-based genome editing technologies have revolutionized genome engineering [31]. These technologies have been widely employed for genome editing in all kingdoms, including animals [32], plants [33], and microbes [34]. Unlike Cre and I-SceI nuclease-based genome editing, CRISPR/Cas-based technology does not require the pre-integration of a unique enzyme recognition sequence into the genome, but employs a transcribed synthetic guide RNA (sgRNA, the chimera of crRNA and tracrRNA), or only crRNA, to direct Cas proteins to any site on the genome [35]. Of the diverse known Cas endonucleases, the Streptococcus pyogenes-derived Cas9, which belongs to Class 2 type II, is the most widely used in Streptomyces [36]. Recently, Cpf1 (also known as Cas12a) derived from Francisella novicida, which belongs to Class 2 type V, has also been used for engineering streptomycetes [37,38]. Compared to other genome editing technologies, the CRISPR/Cas-assisted technology has several advantages, such as higher efficiency, ease of operation, and lower operation time. The CRISPR/Cas-based genome editing technology has markedly promoted the genetic engineering of streptomycetes [39]. Additionally, several CRISPR/Cas-derived technologies, such as the CRISPR interference (CRISPRi)-mediated gene repression tool based on dCas9 (a nuclease-deficient Cas9 with two mutations of D10A and H840A) [40] or ddCpf1 (a nuclease-deficient Cpf1 with the mutation of E1006A) [37] and the base editors (BEs) for targeted base mutagenesis based on dCas9 or Cas9n (a nickase version of Cas9, with the mutation of D10A) have recently been developed [41,42]. They are powerful supplements to the CRISPR/Cas-based genome editing toolbox in Streptomyces. A brief description of the CRISPR/Cas-based technologies for genome editing and gene repression in Streptomyces is presented in Figure 2 and Table 1. 
Table 1. The clustered regularly interspaced palindrome repeat (CRISPR)/CRISPR-associated protein (Cas) system-based plasmids developed for genome editing in Streptomyces.

\begin{tabular}{|c|c|c|c|c|c|c|c|c|c|}
\hline Editing Plasmids & Replicons & Cas Proteins & Origins & $\begin{array}{l}\text { Promoters of } \\
\text { Cas Proteins }\end{array}$ & $\begin{array}{c}\text { Promoters of Guide } \\
\text { RNA }\end{array}$ & $\begin{array}{c}\text { Editing } \\
\text { Efficiency }\end{array}$ & $\begin{array}{l}\text { Other } \\
\text { Features }\end{array}$ & $\begin{array}{c}\text { Addgene Number or Source } \\
\text { of Plasmid Request }\end{array}$ & Reference \\
\hline pCRISPomyces-1 & pSG5 & Cas9 & Streptococcus pyogenes & $\operatorname{rps} L \mathrm{p}(\mathrm{XC})$ & $\begin{array}{l}\text { rpsLp(CF)-tracrRNA } \\
\text { gapdhp(EL)-crRNA }\end{array}$ & $21-25 \%$ & - & 61736 & [43] \\
\hline pCRISPomyces-2 & pSG5 & Cas9 & Streptococcus pyogenes & $r p s L \mathrm{p}(\mathrm{XC})$ & gapdhp(EL)-sgRNA & $67-100 \%$ & - & 61737 & [43] \\
\hline pKCcas9dO & pSG5 & Cas9 & Streptococcus pyogenes & $\operatorname{tip} A p$ & j23119-sgRNA & $29-100 \%$ & - & 62552 & [44] \\
\hline pCRISPR-Cas9 & pSG5 & Cas9 & Streptococcus pyogenes & tipAp & ermEp*-sgRNA & $3-100 \%$ & - & 125686 & [45] \\
\hline pCRISPR-Cas9-ScaligD & pSG5 & Cas9 & Streptococcus pyogenes & tipAp & ermEp*-sgRNA & $69-77 \%$ & LigD & 125688 & [45] \\
\hline pCRISPR-dCas9 & pSG5 & dCas9 & Streptococcus pyogenes & tipAp & ermEp* ${ }^{*}$ sgRNA & ND & - & 125687 & [45] \\
\hline pWHU2653 & pIJ101 & Cas9 & Streptococcus pyogenes & $a a c(3) I V \mathrm{p}$ & ermE $\mathrm{p}^{*}$-sgRNA & $93-99 \%$ & $\operatorname{CodA}(\mathrm{sm})$ & Yuhui Sun group & [46] \\
\hline pMWCas9 & pIJ101 & Cas9 & Streptococcus pyogenes & tipAp & ermEp*-sgRNA & ND & $\operatorname{CodA}(\mathrm{sm})$ & Xudong Qu group & [47] \\
\hline pQS-gusA & pIJ101 & Cas9 & Streptococcus pyogenes & tipAp & ermEp ${ }^{*}$-sgRNA & $100 \%$ & GusA & Chengzhang Fu group & [48] \\
\hline pQS-idgS & pIJ101 & Cas9 & Streptococcus pyogenes & $\operatorname{tip} A p$ & ermEp*-sgRNA & $100 \%$ & IdgS & Chengzhang Fu group & [48] \\
\hline pWHU2653-TRMA & pIJ101 & Cas9 & Streptococcus pyogenes & $\operatorname{tip} A \mathrm{p}$ & ermEp*-sgRNA & $8.3-80 \%$ & AtpD & Xuming Mao group & [49] \\
\hline pKC1139-TRMA & pSG5 & Cas9 & Streptococcus pyogenes & tipAp & ermEp*-sgRNA & $8.3-80 \%$ & AtpD & Xuming Mao group & [49] \\
\hline pKCCpf1 & pSG5 & Cpf1 & Francisella novicida & ermEp* & $k a s O \mathrm{p}^{*}$-crRNA & $75-95 \%$ & - & Yinhua Lu group & [37] \\
\hline pKCCpf1-MsmE & pSG5 & Cpf1 & Francisella novicida & ermE $\mathrm{p}^{*}$ & kasOp*-crRNA & $10-56.7 \%$ & LigD, Ku & Yinhua Lu group & [37] \\
\hline pSETddCpf1 & - & ddCpf1 & Francisella novicida & ermEp* & kasOp*-crRNA & $11.8-95.2 \%$ & - & Yinhua Lu group & [37] \\
\hline pCRISPomyces-Sth1Cas9 & pSG5 & Cas9 & Streptococcus thermophilus & $r p s L \mathrm{p}(\mathrm{XC})$ & gapdhp(EL)-sgRNA & $100 \%$ & - & 129552 & {$[38]$} \\
\hline pCRISPomyces-SaCas9 & pSG5 & Cas9 & Staphylococcus aureus & $r p s L \mathrm{p}(\mathrm{XC})$ & gapdhp(EL)-sgRNA & $87-100 \%$ & - & 129553 & [38] \\
\hline pCRISPomyces-FnCpf1 & pSG5 & Cpf1 & Francisella novicida & $r p s L \mathrm{p}(\mathrm{XC})$ & gapdhp(EL)-crRNA & $87-100 \%$ & - & 129554 & [38] \\
\hline pSET-dCas9 & - & dCas9 & Streptococcus pyogenes & ermEp* & - & - & - & 110183 & [40] \\
\hline pSET-dCas9-actII-4-NT-S1 & - & dCas9 & Streptococcus pyogenes & ermEp* & j23119-sgRNA & $68-99 \%$ & - & 110185 & [40] \\
\hline pCRISPR-cBEST & pSG5 & Cas9n & Streptococcus pyogenes & tipAp & ermE p $^{*}$ sgRNA & $0-100 \%$ & rAPOBEC1 & 125689 & [41] \\
\hline pCRISPR-aBEST & pSG5 & Cas9n & Streptococcus pyogenes & tip $A p$ & ermEp*-sgRNA & $0-100 \%$ & ecTadA & 131464 & [41] \\
\hline pKC-dCas9-CDA-UL ${ }_{s t r}$ & pSG5 & dCas9 & Streptococcus pyogenes & tipAp & j23119-sgRNA & $15-100 \%$ & PmCDA1 & Yinhua Lu group & [42] \\
\hline
\end{tabular}




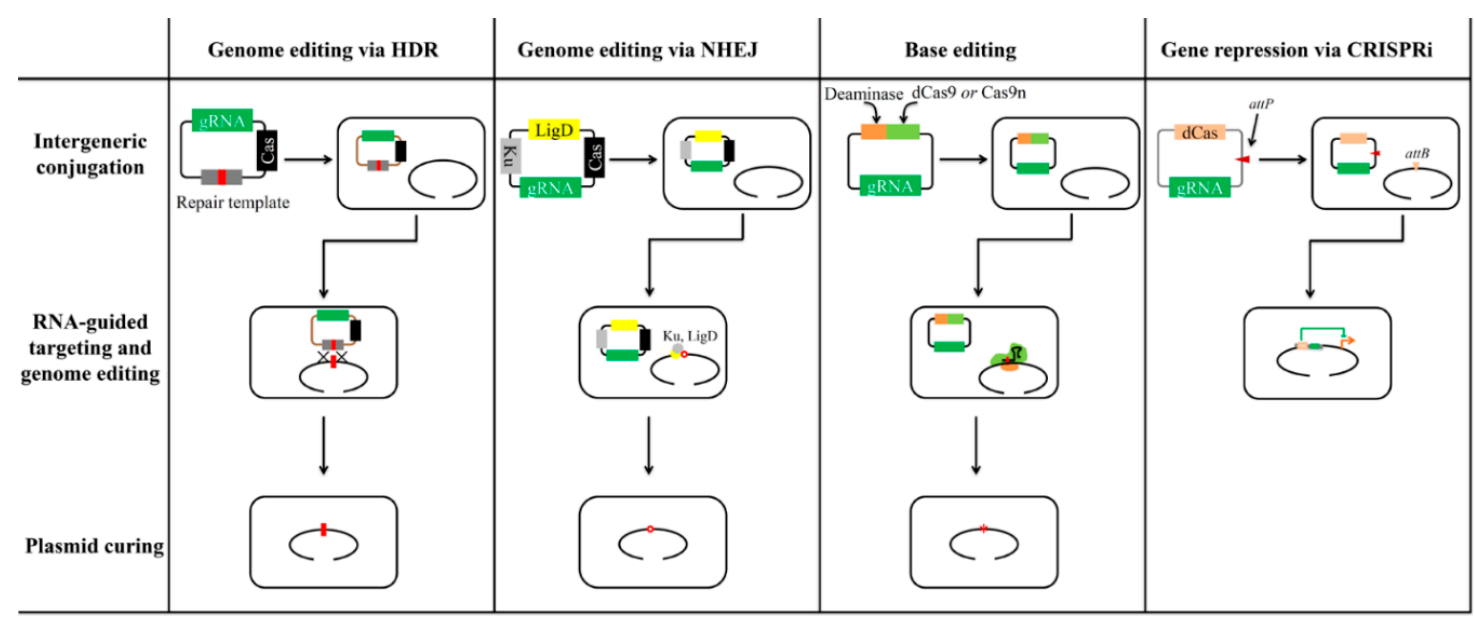

Figure 2. A brief illustration of the CRISPR/Cas system-based technologies for genome editing in Streptomyces. Column 1: Genome editing by homology-directed repair (HDR). An editing plasmid with the expression cassettes of the Cas endonuclease and a small guide RNA (gRNA), and containing homologous repair template is introduced into Streptomyces by intergeneric conjugation. The Cas nuclease cleaves the target site on the genome with the help of gRNA, resulting in double-strand break (DSB). The DSB is repaired by HDR in the presence of homologous repair template. Then, specific mutations, such as deletions, insertions, and point mutations, are introduced. After plasmid curing, the edited strain could be subject to next round genome editing. Column 2: Genome editing by non-homologous end joining (NHEJ). An editing plasmid containing the expression cassettes of the Cas endonuclease, a small gRNA, and the $k u / l i g D$ genes is introduced into Streptomyces by intergeneric conjugation. After RNA-guided DNA cleavage by the Cas nuclease, the DSB is repaired by the action of LigD and $\mathrm{Ku}$. Subsequently, the editing plasmid is cured to facilitate next round genome editing. Column 3: Base editor. The gene encoding a deaminase is fused with the $d \operatorname{Cas} 9$ or $\operatorname{Cas} 9 n$ gene to induce base substitutions. After introduction of the editing plasmid into Streptomyces by intergeneric conjugation, the fusion protein could achieve RNA-guided base editing within a specific target window. After plasmid curing, the edited strain could be subject to next round genome editing. Column 4: Gene repression by CIRPSRi. An integrative plasmid with the expression cassettes of the nuclease-deficient $\mathrm{Cas}(\mathrm{dCas})$ gene and gRNA is introduced into Streptomyces by conjugation. After site-specific recombination, the plasmid is integrated into the genome. dCas/gRNA complex could repress the transcription of target genes by interfering with transcriptional initiation or elongation.

\subsection{Cas9-Based Genome Editing}

In 2015, four research groups successively developed CRISPR/Cas9-based genome editing tools, which enabled efficient and rapid genetic manipulation, including deletions of single genes or gene clusters, simultaneous deletions of two genes or gene clusters, and point mutations in Streptomyces [43-46]. Cobb et al. generated two sets of CRISPR/Cas9 genome editing systems, namely pCRISPomyces- 1 and pCRISPomyces-2, and achieved the precise deletion of various DNA sizes (ranging from $20 \mathrm{bp}-31.4 \mathrm{~kb}$ ) (including individual genes, double genes simultaneously, and single antibiotic BGCs) with efficiency of $21-100 \%$ by homology-directed repair (HDR) in three different Streptomyces species [43]. Later, they applied this CRISPR/Cas9 technology to perform promoter knock-in for the activation of silent BGCs of different classes in five native Streptomyces hosts and achieved the identification of unique metabolites, including a novel pentangular type II polyketide in Streptomyces viridochromogenes [15]. We developed a similar CRISPR/Cas9-based editing system, namely, $\mathrm{pKC}$ cas9dO, in $S$. coelicolor. This system was applied for genetic engineering at different levels with high efficiency ranging from 60-100\%, including deletion of individual genes (actII-orf4, redD, and glnR) and single antibiotic BGCs (with a size of 21.3, 31.6 and $82.8 \mathrm{~kb}$, respectively) [44]. Furthermore, we achieved simultaneous deletion of two genes (actII-orf4 and redD) and two BGCs (21.3 and $31.6 \mathrm{~kb}$ ) 
with an efficiency of $54 \%$ and $45 \%$, respectively. Additionally, this system was applied to introduce point mutations into the rpsL gene with an efficiency of $64 \%$. Around the same time, Tong et al. established the CRISPR/Cas9 editing system (pCRISPR-Cas9) for precise deletion of individual genes and multiple genes by HDR in S. coelicolor [45]. Moreover, they reconstituted the non-homologous end joining (NHEJ) repair pathway by introducing the ligD gene from Streptomyces carneus and generated the editing plasmid pCRISPR-Cas9-ScaligD. This tool acheived high-efficiency inactivation of target genes in the absence of homologous DNA templates. All these genome editing systems, including pCRISPomyces-1, pCRISPomyces-2, pKCcas9dO, and pCRISPR-Cas9, are developed based on plasmids harboring pSG5, a temperature-sensitive replicon [50]. To obtain plasmid-free mutants and perform multiple rounds of genome editing, the editing plasmids must be cured by at least two or three passages at a high temperature (e.g., $37^{\circ} \mathrm{C}$ ), which is a time-consuming process, especially for slow-growing streptomycetes. Zeng et al. developed the CRISPR/Cas9 editing system pWHU2653 based on a plasmid with pIJ101, a segregationally unstable replicon. Additionally, the counter-selection marker CodA(sm) (the D314A mutant of cytosine deaminase CodA) was introduced [46], which converts 5-fluorocytosine (5-FC) to the toxic compound 5-fluorouracil (5-FU). The introduction of CodA(sm) simplified plasmid curing. The efficiency of plasmid curing can be improved to $95 \%$, which is higher than that of the pIJ101-derived editing plasmid without CodA(sm) (only 39\%).

Recently, two modified CRISPR/Cas9 genome editing tools from pWHU2653 have been developed. Mo et al. constructed the editing system pMWCas9 based on pWHU2653 by replacing the ermEp* promoter of Cas 9 with the thiostrepton-inducible promoter tipAp, which can significantly enhance the DNA transformation efficiency [47]. Importantly, pMWCas9 was successfully used to delete highly repetitive DNA sequences, such as the eryAIII gene from erythromycin polyketide synthase (PKS) in Saccharopolyspora erythraea. In contrast, the pCRISPR-Cas9 editing tool failed to delete the target gene, which may be caused by the unpredicted DNA recombination resulting from the pSG5 replicon [51]. Wang et al. developed an updated dual-functional chromogenic-screening CRISPR/Cas9 tool (pQS-gusA and pQS-idgS) based on pWHU2653 by replacing the counter-selection marker CodA(sm) with two reporter systems, GusA and IdgS [48]. These two reporter systems improved the efficiency of both genome editing and plasmid curing up to $100 \%$ after chromogenic screening and further simplified the plasmid curing process. This editing system was also successfully applied for efficient genetic manipulation of the genetically recalcitrant and slow-growing rare actinomycete strain Verrucosispora sp. MS100137. This genome editing system achieved an efficiency of up to 100\% for the deletion of both the carotenoid BGC $(5.5 \mathrm{~kb})$ and the abyssomicin BGC $(61 \mathrm{~kb})$. In addition, a modified editing tool was developed from the pCRISPR-Cas9 plasmid by introducing both the $S$. carneus-derived ligD (required for NHEJ) and a homology template (required for HDR) into one plasmid, thereby allowing either DNA repair pathway to occur [13]. By designing one sgRNA harboring a conserved protospacer sequence targeting a BGC gene of interest, this system achieved the deletion of genes in the BGCs responsible for the biosynthesis of streptothricin or streptomycin (two of the most frequently rediscovered antibiotics) in 11 actinomycete strains with an efficiency of up to $100 \%$. CRISPR/Cas9-mediated inactivation of commonly found BGCs allows the mining of a greater proportion of actinimycete strain collections (many of them are streptomycetes) for new NPs.

A major challenge for CRISPR/Cas9-mediated genome editing is the high toxicity of Cas9 to the host derived from the off-target DNA cleavage and non-target DNA binding in the absence of sgRNAs, which hampers its application in streptomycetes with low DNA transformation efficiency. To address this issue, Wang et al. established a modified CRISPR/Cas9 system based on pWHU2653 (named pWHU2653-TRMA) by modulating Cas9 activity at multiple levels: (i) using the inducible promoter tipAp to regulate the expression of Cas9 instead of the strong constitutive promoter (at the transcriptional level); (ii) introducing the theophylline-inducible riboswitch and Mag-based blue light-inducible reconstitution system to regulate Cas9 activity at the translational and protein levels, respectively [49]. Additionally, as DSB repair is an ATP-dependent process, the gene encoding the ATP synthase $\beta$-subunit AtpD was introduced for overexpression to increase the editing efficiency. In $S$. 
coelicolor, under non-induction conditions, triple controls of Cas 9 could markedly reduce its toxicity and increase DNA transformation efficiency by over 250-fold when compared to pWHU2653. As the counter-selection marker $\operatorname{CodA}(\mathrm{sm})$ is not suitable for Streptomyces with high resistance, they also developed a similar system pKC1139-TRMA based on pKC1139. The pKC1139-TRMA was used to achieve individual deletion of actII-orf4 and redD in S. coelicolor with efficiencies ranging from $35 \%$ to $80 \%$ after simultaneous induction with thiostrepton, theophylline, and blue light for Cas 9 activity reconstitution. The pWHU2653-TRMA and pKC1139-TRMA editing systems can achieve the uncoupling of DNA transformation and Cas9-mediated DNA cleavage, which will markedly improve the genetic engineering of Streptomyces species with low DNA transformation efficiency.

The genomes of Streptomyces strains contain many multicopy genes and mobile genetic elements. The editing of these genes is very difficult because they have identical or highly similar DNA sequences. To address this issue, Najah et al. developed a generic two-step CRISPR/Cas9 editing tool [52], which is similar to I-SceI meganuclease-assisted genome editing technology [28]. First, a non-replicative plasmid (bait DNA) containing the homologous arms flanking the target gene was integrated into the genome by single-crossover recombination. Next, another plasmid was introduced to express Cas9 and sgRNA to cleave the bait DNA (such as the antibiotic-resistance gene), which induced the double-crossover recombination events. The native copies of two xenogeneic silencers $l s r 2$ paralogs were deleted in Streptomyces ambofaciens using this technology. This approach can be widely used for specifically editing one copy of multicopy genes, as well as for the exploration of gene essentiality in Streptomyces.

\subsection{Cpf1-Assisted Genome Editing}

The CRISPR/Cas9-based genome editing tools presented above have some limitations that must be addressed. For instance, a G-rich protospacer-adjacent motif (PAM) sequence ( $5^{\prime}$-NGG-3') is required for target sequence recognition by Cas9. Due to the high GC content of the Streptomyces genome $(>70 \%)$, the PAM sequence is frequently distributed across Streptomyces genomes (e.g., 260 targets per $1000 \mathrm{bp}$ in S. coelicolor) [37]. However, the PAM sequence may not be present in the AT-rich DNA regions. Furthermore, to implement multiplex gene editing, the process of plasmid construction for independent transcription of multiple sgRNAs (each has its promoter and terminator) and the introduction of homologous DNA templates for DSB repair is complex and time-consuming. To address these limitations, Cpf1 from Francisella novicida (FnCpf1) that recognizes T-rich PAM sequences ( $5^{\prime}$-TTV-3) has been developed for Streptomyces genome engineering by our group [37]. Cpf1 has RNase activity for pre-crRNA processing and the expression of multiple guide crRNAs requires only one promoter, which is an advantage for multiplex genome editing [53]. We achieved the precise deletion of single genes or double genes simultaneously based on HDR with high-efficiency (75-95\%) in S. coelicolor. Furthermore, we introduced the codon-optimized Mycobacterium smegmatis $l i g D$ and $k u$ genes to reconstitute the NHEJ pathway. The inactivation of target genes or gene clusters by NHEJ-assisted DSB repair can result in random-sized DNA deletions. Further, we observed that Cas9 from S. pyogenes and Cpf1 from F. novicida exhibited different suitability in the seven tested Streptomyces species. Using FnCpf1, we achieved gene deletion in the 5-oxomilbemycin A3/A4-producing strain Streptomyces hygroscopicus SIPI-KF, which cannot be edited by Cas9 due to its high toxicity. Similarly, as many Streptomyces strains cannot be edited by S. pyogenes Cas9, Yeo et al. tested several alternative CRISPR-Cas systems based on the pCRISPomyces-2 system [38]. They demonstrated that Cas9 from Streptococcus thermophilus CRISPR1 (Sth1Cas9, PAM: NNAGAA and NNGGAA), Cas9 from Staphylococcus aureus (SaCas9, PAM: NNGRRT), and Cpf1 from F. novicida (FnCpf1) are functional in multiple streptomycetes, which enables efficient HDR-mediated DNA knock-in and gene deletion. The Cpf1- and alternative Cas9-assisted genome editing technologies can efficiently edit strains that cannot be edited by Cas9 from S. pyogenes, such as Streptomyces sp. NRRL S-244. Therefore, they are a good complement to the current Cas9-based tools. Hence, a diverse CRISPR/Cas toolbox will markedly facilitate NP discovery and overproduction in Streptomyces as well as other actinomycetes. 


\section{3. dCas-Based Transcriptional Repression (CRISPRi)}

The CRIPSRi tool based on the nuclease-defective Cas nuclease (such as dCas9 and ddCpf1) can be employed for efficient gene repression by hindering transcription initiation and elongation, which has been demonstrated to be a powerful tool for functional genome research and metabolic engineering in bacteria [54]. Recently, three different CRISPRi tools have been developed in Streptomyces. Tong et al. developed an inducible CRISPRi based on genome editing system pCRISPR-Cas 9 by replacing Cas9 with dCas9, which enabled efficient repression of single genes upon induction [45]. In this CRISPRi system, the replicative plasmid pGM1190, which harbors a temperature-sensitive replicon pSG5, was used to express the dCas9/sgRNA complex and the dCas9 gene is regulated by the thiostrepton-inducible promoter (tipAp). Our research group developed two CRISPRi tools in S. coelicolor based on dCas9 [40] and ddCpf1 [37]. In these two systems, pSET152, an integrative plasmid, was used for the expression of the dCas9/sgRNAs or ddCpf1/crRNAs complex and both $\mathrm{dCas} 9$ and sgRNAs or ddCpf1 and crRNAs were designed to be regulated by constitutive promoters. Using these two CRISPRi systems, we achieved simultaneous repression of up to four genes at high efficiency. Compared to the inducible CRISPRi tool based on the replicative plasmid (e.g., pGM1190), the latter two systems may have two advantages. First, their repression effects are likely to be stable as they are integrated into the genome. Second, they have wider application, because the efficiency of pSET152 transformation is higher than that of the replicative plasmids. Meanwhile the $\phi C 31$ att $B$ site for pSET152 integration is present on the genome of all Streptomyces strains (whose genome sequences are available so far) [55]. It is important to note that simultaneous repression of multiple targets using the dCas9-based system involves a time-consuming procedure to construct multiple sgRNA expression cassettes with independent promoters and terminators. In contrast, only a single customized CRISPR array with one promoter is required owing to the pre-crRNA processing ability of ddCpf1, which is time-saving and convenient. Therefore, the ddCpf1-based CRISPRi system has an advantage over dCas9-based systems for multiplexed gene repression.

\subsection{Base Editors Based on the Cas9 Variants (dCas9 or Cas9n)}

CRISPR-guided BEs are emerging genome editing technologies developed in recent years, which enable efficient targeted single-nucleotide-resolution DNA mutagenesis in the genome and are novel powerful tools for genome editing in mammalian cells [56], animals [57], plants [58], and bacteria [59]. The basic principle of BEs is to fuse a Cas9 variant, such as dCas9 (D10A and $\mathrm{H} 840 \mathrm{~A}$ ) or Cas9n (D10A), with a base deaminase to deaminate the exocyclic amine of the target bases, thereby leading to base substitutions [60]. In contrast to the CRISPR/Cas-based genome editing tools mentioned above, BEs do not create DNA DSBs and do not rely on cellular HDR or NHEJ DNA repair pathways. Therefore, it reduces the by-products related to DSBs, such as small insertions or deletions (indels) [61]. Currently, the following two kinds of DNA BEs have been established: cytosine base editors (CBEs) and adenine base editors (ABEs) [62]. CBE can convert cytidine (C) to thymidine (T), while ABE can be used to convert adenosine (A) to guanosine (G). Both types of BEs have been developed for genome editing in Streptomyces [41,42].

Tong et al. developed two base editing systems, CRISPR-cBEST (belonging to CBE) and CRISPR-aBEST (belonging to ABE), by fusing rat APOBEC1 (rAPOBEC1) cytidine deaminase and the adenosine deaminase ecTadA to the $\mathrm{N}$-terminus of the codon-optimized Cas9n [41]. To inhibit the activity of the uracil-DNA glycosylase (UDG) and increase the editing efficiency, a codon-optimized uracil glycosylase inhibitor (UGI) from Bacillus phage AR9 is linked to the C-terminus of Cas9n in CRISPR-cBEST. In S. coelicolor, CRISPR-cBEST converted cytidine to thymidine within a 7-base target window ( -11 to $-17 \mathrm{bp}$ upstream of the PAM sequence) with frequencies up to $100 \%$. CRISPR-aBEST can convert adenosine to guanosine within a 6-base target window, -12 to $-17 \mathrm{bp}$ upstream of PAM. The editing of CRISPR-cBEST follows the priority of TC $>$ CC $>$ AC $>$ GC, while that of CRISPR-aBEST follows the priority of TA > GA > AA > CA. Compared to CRISPR-aBEST, CRISPR-cBEST had higher editing efficiency and off-target effects. Using CRISPR-cBEST, the authors achieved the precise 
introduction of STOP codons into the designed DNA locations at high frequencies of $60 \%$ to $100 \%$ in Streptomyces griseofuscus and simultaneous targeted mutagenesis of two identical gene copies of the gene kirN in Streptomyces collinus Tü365. Finally, by introducing the Csy4-based RNA processing system between sgRNA for the expression of multiple sgRNAs with one promoter and terminator, the authors achieved simultaneous editing of three different sites at frequencies up to $100 \%$. Recently, our group has also developed a CBE system dCas9-CDA-UL ${ }_{s t r}$ derived from the CBE dCas9-CDA-UL established in E. coli [63], which comprises dCas9, PmCDA1 (an activation-induced cytidine deaminase (AID) ortholog from Petromyzon marinus), UGI, and the degradation tag (LVA) [42]. Using dCas9-CDA-UL str $_{\text {, }}$ we achieved single-, double-, and triple-point mutations (cytidine to thymidine) at target sites in S. coelicolor with high efficiencies of up to $100 \%, 60 \%$, and $20 \%$, respectively. This CBE was also applicable for highly efficient base editing in the industrial strain, Streptomyces rapamycinicus. Compared to CRISPR-cBEST, which has a 7-base editing window, dCas9-CDA-UL str $_{\text {has }}$ a 5-base editing window, -16 to $-20 \mathrm{bp}$ upstream of the PAM sequence. Moreover, dCas9-CDA-UL $\mathrm{L}_{\text {str }}$ had higher editing efficiency (70-100\%) for cytidines preceded by guanosines than rAPOBEC1-derived CRISPR-cBEST $(0-60 \%)$, which is an advantage for base editing in streptomycetes with high GC contents in their genomes. The development of BEs provides alternative and powerful tools for genome editing in Streptomces. In particular, four amino acid codons, namely, Arg (CGA), Gln (CAA and CAG), and Trp codons (TGG, target $C$ in the non-coding strand) can be efficiently mutated to STOP codons (TGA, TAA, and TAG) using CBEs, which results in the inactivation of gene function [64]. Compared to HDR-mediated gene deletion, CBE-based introduction of STOP codons is time- and labor-saving without the cloning of the repair templates. Therefore, we believe that CBEs will significantly facilitate functional genome research and metabolic engineering-based strain improvement in streptomycetes, especially those with weak HR ability.

\section{Conclusions and Perspectives}

The activation of silent BGCs in either native or heterologous Streptomyces hosts for NPs discovery normally require extensively genetic manipulation. Therefore, it is critical to develop high-efficiency genome editing tools. Recently, the development and application of the CRISPR/Cas system (particularly, S. pyogenes Cas9)-assisted genome editing technologies have markedly improved Streptomyces genome engineering, which has facilitated genome mining for novel NP discovery and strain improvement for NP overproduction. However, their application for genome editing requires a high DNA transformation frequency due to the toxic effect of S. pyogenes Cas9, as well as the relatively large DNA size of the editing plasmids (normally $>10 \mathrm{~kb}$ ). Therefore, their application is a big challenge in streptomycetes with low DNA transformation ability. To address this issue, researchers have recently adopted two strategies: (i) uncoupling the process between DNA transformation and Cas9-based DNA cleavage by inducing Cas9 expression at the transcription, translation, and protein levels [49]; (ii) using alternative CRSIPR/Cas systems, such as S. thermophilus Cas9, S. aureus Cas9, and F. novicida Cpf1, which have less lethal effects $[37,38]$. However, owing to the infrequent distribution of PAMs in the Streptomyces genome recognized by these alternative Cas nucleases, they may not be as widely used as $S$. pyogenes Cas9. In the future, the following two strategies may be employed to address the toxicity of Cas9.

(i) Developing the Cas9 nickase (Cas9n)-based genome editing technology. Cas9n only causes single-stranded DNA breaks, which can mitigate the lethal effects of Cas9. Currently, Cas9n has been adopted for the development of efficient and precise genome editing tools in several bacteria, such as Clostridium cellulolyticum [65], Pseudomonas putida [66], Escherichia coli [67], Lactobacillus casei [68], and Bacillus licheniformis [69]. However, Cas9n has not been adopted for Streptomyces genome editing. We believe that the Cas9n-assisted genome editing technology may be suitable for engineering streptomycetes with low DNA transformation efficiency.

(ii) Developing genome editing tools based on endogenous CRISPR/Cas systems. CRISPR/Cas systems are reported in approximately $45 \%$ of bacterial genomes, including streptomycetes, which are a rich source for the development of endogenous CRISPR/Cas nuclease-based genome editing 
tools [30]. In addition to mitigating the toxicity of heterologous Cas nucleases by harnessing endogenous CRISPR/Cas systems, the DNA transformation frequency can be improved as the size of the editing plasmid is approximately $4.2 \mathrm{~kb}$ (the size of the $S$. pyogenes cas 9 gene) smaller than that based on heterologous Cas9. Therefore, endogenous CRISPR/Cas machinery provides a superior foundation for genome editing by precluding heterologous cas 9 expression. For example, Pyne et al. reported the detailed characterization of a functional native Type I-B CRISPR/Cas system in Clostridium pasteurianum [70]. This CRISPR/Cas system was repurposed for precise markerless genome editing. Compared to the widely employed S. pyogenes type II CRISPR-Cas9 system that generated $25 \%$ of the total yield of edited cells, the endogenous Cas system-based tool could achieve gene deletion with an efficiency of $100 \%$ by only introducing a synthetic crRNA array and templates for DSB repair. In addition to C. pasteurianum, endogenous CRISPR-Cas systems have been repurposed for genome editing in various bacteria, including Escherichia coli [71], Pectobacterium atrosepticum [72], Streptococcus thermophilus [73], Streptococcus mutans [74], Lactobacillus crispatus [75], and Clostridium tyrobutyricum [76]. Recently, Zhang et al. comparatively analyzed the wide distribution of native CRISPR/Cas system in the genus Streptomyces [77]. The homologous cas gene clusters were searched in the genome sequences of 46 Streptomyces species harboring the CRISPR loci. The analysis revealed that 26 strains contained 29 cas gene clusters. Of these, 26 clusters belonged to the type I-E subtype, two clusters belonged to I-C types, and one cluster belonged to the I-U subtype. Additionally, Qiu et al. identified an active type I-E CRISPR-Cas system with a completely conserved PAM sequence (5'-AAG-3') in the industrial strain S. avermitilis [78]. By repurposing the CRISPR/Cas system, they achieved strain protection from the infection of phages with target protospacers. In the future, endogenous CRISPR/Cas systems in streptomycetes can be readily applied for genome editing by only delivering an engineered synthetic CRISPR array or a small RNA guide in streptomycetes with a limited activity of heterologous CRISPR/Cas systems. However, the specific PAM sequences and the activity of the endogenous Cas nucleases must be fully characterized before repurposing them for genome editing, which can be achieved by plasmid interference assays combined with bioinformatics analysis (e.g., CRISPRTarget) [79].

Funding: This work was supported by the National Key R\&D Program (2019YFA0905400), the National Natural Science Foundation of China (31970083 and 31400083), the National Mega-project for Innovative Drugs (2018ZX09711001-006-012), the Key R\&D Plan of Zhenjiang City (NY2018015), and the Key R\&D Plan of Dantu District (NY2017001).

Conflicts of Interest: There are no conflicts of interest to declare.

\section{References}

1. Mast, Y.; Weber, T.; Gölz, M.; Ort-Winklbauer, R.; Gondran, A.; Wohlleben, W.; Schinko, E. Characterization of the 'pristinamycin supercluster'of Streptomyces pristinaespiralis. Microb. Biotechnol. 2011, 4, 192-206. [CrossRef] [PubMed]

2. Baltz, R.H. Daptomycin: mechanisms of action and resistance, and biosynthetic engineering. Curr. Opin. Chem. Biol. 2009, 13, 144-151. [CrossRef] [PubMed]

3. Yoo, Y.J.; Kim, H.; Park, S.R.; Yoon, Y.J. An overview of rapamycin: From discovery to future perspectives. J. Ind. Microbiol. Biotechnol. 2017, 44, 537-553. [CrossRef] [PubMed]

4. Barreiro, C.; Martínez-Castro, M. Trends in the biosynthesis and production of the immunosuppressant tacrolimus (FK506). Appl. Microbiol. Biotechnol. 2014, 98, 497-507. [CrossRef] [PubMed]

5. Yoon, Y.; Kim, E.-S.; Hwang, Y.-S.; Choi, C.-Y. Avermectin: biochemical and molecular basis of its biosynthesis and regulation. Appl. Microbiol. Biotechnol. 2004, 63, 626-634. [CrossRef]

6. Li, J.-S.; Du, M.-N.; Zhang, H.; Zhang, J.; Zhang, S.-Y.; Wang, H.-Y.; Chen, A.-L.; Wang, J.-D.; Xiang, W.-S. New milbemycin metabolites from the genetically engineered strain Streptomyces bingchenggensis BCJ60. Nat. Prod. Res. 2017, 31, 780-784. [CrossRef]

7. Vasanthakumar, A.; Kattusamy, K.; Prasad, R. Regulation of daunorubicin biosynthesis in Streptomyces peucetius-feed forward and feedback transcriptional control. J. Basic Microbiol. 2013, 53, 636-644. [CrossRef] 
8. Shen, B.; Du, L.; Sanchez, C.; Edwards, D.; Chen, M.; Murrell, J. The biosynthetic gene cluster for the anticancer drug bleomycin from Streptomyces verticillus ATCC15003 as a model for hybrid peptide-polyketide natural product biosynthesis. J. Ind. Microbiol. Biotechnol. 2001, 27, 378-385. [CrossRef]

9. Tulp, M.; Bohlin, L. Rediscovery of known natural compounds: nuisance or goldmine? Bioorg. Med. Chem. 2005, 13, 5274-5282. [CrossRef]

10. Baltz, R.H. Gifted microbes for genome mining and natural product discovery. J. Ind. Microbiol. Biotechnol. 2017, 44, 573-588. [CrossRef]

11. Onaka, H. Novel antibiotic screening methods to awaken silent or cryptic secondary metabolic pathways in actinomycetes. J. Antibiot. 2017, 70, 865-870. [CrossRef] [PubMed]

12. Rutledge, P.J.; Challis, G.L. Discovery of microbial natural products by activation of silent biosynthetic gene clusters. Nat. Rev. Microbiol. 2015, 13, 509-523. [CrossRef] [PubMed]

13. Culp, E.J.; Yim, G.; Waglechner, N.; Wang, W.; Pawlowski, A.C.; Wright, G.D. Hidden antibiotics in actinomycetes can be identified by inactivation of gene clusters for common antibiotics. Nat. Biotechnol. 2019, 37, 1149-1154. [CrossRef] [PubMed]

14. Sidda, J.D.; Song, L.; Poon, V.; Al-Bassam, M.; Lazos, O.; Buttner, M.J.; Challis, G.L.; Corre, C. Discovery of a family of $\gamma$-aminobutyrate ureas via rational derepression of a silent bacterial gene cluster. Chem. Sci. 2014, 5, 86-89. [CrossRef]

15. Zhang, M.M.; Wong, F.T.; Wang, Y.; Luo, S.; Lim, Y.H.; Heng, E.; Yeo, W.L.; Cobb, R.E.; Enghiad, B.; Ang, E.L. CRISPR-Cas9 strategy for activation of silent Streptomyces biosynthetic gene clusters. Nat. Chem. Biol. 2017, 13, 607-609. [CrossRef]

16. Laureti, L.; Song, L.; Huang, S.; Corre, C.; Leblond, P.; Challis, G.L.; Aigle, B. Identification of a bioactive 51-membered macrolide complex by activation of a silent polyketide synthase in Streptomyces ambofaciens. Proc. Natl. Acad. Sci. USA 2011, 108, 6258-6263. [CrossRef]

17. Nepal, K.K.; Wang, G. Streptomycetes: surrogate hosts for the genetic manipulation of biosynthetic gene clusters and production of natural products. Biotechnol. Adv. 2019, 37, 1-20. [CrossRef]

18. Kieser, T.; Bibb, M.J.; Buttner, M.J.; Chater, K.F.; Hopwood, D.A. Practical Streptomyces Genetics; The John Innes Foundation: Norwich, UK, 2000.

19. Tao, W.; Yang, A.; Deng, Z.; Sun, Y. CRISPR/Cas9-based editing of Streptomyces for discovery, characterization, and production of natural products. Front. Microbiol. 2018, 9, 1660. [CrossRef]

20. Datsenko, K.A.; Wanner, B.L. One-step inactivation of chromosomal genes in Escherichia coli K-12 using PCR products. Proc. Natl. Acad. Sci. USA 2000, 97, 6640-6645. [CrossRef]

21. Murphy, K.C. Phage recombinases and their applications. Adv. Virus Res. 2012, 83, 367-414.

22. Gust, B.; Challis, G.L.; Fowler, K.; Kieser, T.; Chater, K.F. PCR-targeted Streptomyces gene replacement identifies a protein domain needed for biosynthesis of the sesquiterpene soil odor geosmin. Proc. Natl. Acad. Sci. USA 2003, 100, 1541-1546. [CrossRef] [PubMed]

23. Komatsu, M.; Uchiyama, T.; Ōmura, S.; Cane, D.E.; Ikeda, H. Genome-minimized Streptomyces host for the heterologous expression of secondary metabolism. Proc. Natl. Acad. Sci. USA 2010, 107, 2646-2651. [CrossRef] [PubMed]

24. Bierman, M.; Logan, R.; O’brien, K.; Seno, E.; Rao, R.N.; Schoner, B. Plasmid cloning vectors for the conjugal transfer of DNA from Escherichia coli to Streptomyces spp. Gene 1992, 116, 43-49. [CrossRef]

25. He, Y.; Wang, Z.; Bai, L.; Liang, J.; Zhou, X.; Deng, Z. Two pHZ1358-derivative vectors for efficient gene knockout in Streptomyces. J. Microbiol. Biotechnol. 2010, 20, 678-682. [CrossRef] [PubMed]

26. Monteilhet, C.; Perrin, A.; Thierry, A.; Colleaux, L.; Dujon, B. Purification and characterization of the in vitro activity of I-Sce I, a novel and highly specific endonuclease encoded by a group I intron. Nucleic Acids Res. 1990, 18, 1407-1413. [CrossRef] [PubMed]

27. Choulika, A.; Perrin, A.; Dujon, B.; Nicolas, J.-F. Induction of homologous recombination in mammalian chromosomes by using the I-SceI system of Saccharomyces cerevisiae. Mol. Cell. Biol. 1995, 15, 1968-1973. [CrossRef]

28. Lu, Z.; Xie, P.; Qin, Z. Promotion of markerless deletion of the actinorhodin biosynthetic gene cluster in Streptomyces coelicolor. Acta Biochim. Biophys. Sin. 2010, 42, 717-721. [CrossRef]

29. Fernández-Martínez, L.T.; Bibb, M.J. Use of the Meganuclease I-SceI of Saccharomyces cerevisiae to select for gene deletions in actinomycetes. Sci. Rep. 2014, 4, 7100. [CrossRef] 
30. Makarova, K.S.; Wolf, Y.I.; Alkhnbashi, O.S.; Costa, F.; Shah, S.A.; Saunders, S.J.; Barrangou, R.; Brouns, S.J.; Charpentier, E.; Haft, D.H. An updated evolutionary classification of CRISPR-Cas systems. Nat. Rev. Microbiol. 2015, 13, 722-736. [CrossRef]

31. Wright, A.V.; Nuñez, J.K.; Doudna, J.A. Biology and applications of CRISPR systems: Harnessing nature's toolbox for genome engineering. Cell 2016, 164, 29-44. [CrossRef]

32. Wang, F.; Qi, L.S. Applications of CRISPR genome engineering in cell biology. Trends Cell Biol. 2016, 26, 875-888. [CrossRef] [PubMed]

33. Puchta, H. Applying CRISPR/Cas for genome engineering in plants: the best is yet to come. Curr. Opin. Plant. Biol. 2017, 36, 1-8. [CrossRef] [PubMed]

34. Choi, K.R.; Lee, S.Y. CRISPR technologies for bacterial systems: current achievements and future directions. Biotechnol. Adv. 2016, 34, 1180-1209. [CrossRef] [PubMed]

35. Knott, G.J.; Doudna, J.A. CRISPR-Cas guides the future of genetic engineering. Science 2018, 361, 866-869. [CrossRef]

36. Alberti, F.; Corre, C. Editing streptomycete genomes in the CRISPR/Cas9 age. Nat. Prod. Rep. 2019, 36, 1237-1248. [CrossRef]

37. Li, L.; Wei, K.; Zheng, G.; Liu, X.; Chen, S.; Jiang, W.; Lu, Y. CRISPR-Cpf1-assisted multiplex genome editing and transcriptional repression in Streptomyces. Appl. Environ. Microbiol. 2018, 84, e00827-18. [CrossRef]

38. Yeo, W.L.; Heng, E.; Tan, L.L.; Lim, Y.W.; Lim, Y.H.; Hoon, S.; Zhao, H.; Zhang, M.M.; Wong, F.T. Characterization of Cas proteins for CRISPR-Cas editing in streptomycetes. Biotechnol. Bioeng. 2019, 116, 2330-2338. [CrossRef]

39. Kormanec, J.; Rezuchova, B.; Homerova, D.; Csolleiova, D.; Sevcikova, B.; Novakova, R.; Feckova, L. Recent achievements in the generation of stable genome alterations/mutations in species of the genus Streptomyces. Appl. Microbiol. Biotechnol. 2019, 103, 5463-5482. [CrossRef]

40. Zhao, Y.; Li, L.; Zheng, G.; Jiang, W.; Deng, Z.; Wang, Z.; Lu, Y. CRISPR/dCas9-mediated multiplex gene repression in Streptomyces. Biotechnol. J. 2018, 13, 1800121. [CrossRef]

41. Tong, Y.; Whitford, C.M.; Robertsen, H.L.; Blin, K.; Jørgensen, T.S.; Klitgaard, A.K.; Gren, T.; Jiang, X.; Weber, T.; Lee, S.Y. Highly efficient DSB-free base editing for streptomycetes with CRISPR-BEST. Proc. Natl. Acad. Sci. USA 2019, 116, 20366-20375. [CrossRef]

42. Zhao, Y.; Tian, J.; Zheng, G.; Chen, J.; Sun, C.; Yang, Z.; Zimin, A.A.; Jiang, W.; Deng, Z.; Wang, Z. Multiplex genome editing using a dCas9-cytidine deaminase fusion in Streptomyces. Sci. China Life Sci. 2019, 1-10. [CrossRef] [PubMed]

43. Cobb, R.E.; Wang, Y.; Zhao, H. High-efficiency multiplex genome editing of Streptomyces species using an engineered CRISPR/Cas system. ACS Synth. Biol. 2015, 4, 723-728. [CrossRef] [PubMed]

44. Huang, H.; Zheng, G.; Jiang, W.; Hu, H.; Lu, Y. One-step high-efficiency CRISPR/Cas9-mediated genome editing in Streptomyces. Acta Biochim. Biophys. Sin. 2015, 47, 231-243. [CrossRef] [PubMed]

45. Tong, Y.; Charusanti, P.; Zhang, L.; Weber, T.; Lee, S.Y. CRISPR-Cas9 based engineering of actinomycetal genomes. ACS Synth. Biol. 2015, 4, 1020-1029. [CrossRef] [PubMed]

46. Zeng, H.; Wen, S.; Xu, W.; He, Z.; Zhai, G.; Liu, Y.; Deng, Z.; Sun, Y. Highly efficient editing of the actinorhodin polyketide chain length factor gene in Streptomyces coelicolor M145 using CRISPR/Cas9-CodA (sm) combined system. Appl. Microbiol. Biotechnol. 2015, 99, 10575-10585. [CrossRef]

47. Mo, J.; Wang, S.; Zhang, W.; Li, C.; Deng, Z.; Zhang, L.; Qu, X. Efficient editing DNA regions with high sequence identity in actinomycetal genomes by a CRISPR-Cas9 system. Synth. Syst. Biotechnol. 2019, 4, 86-91. [CrossRef]

48. Wang, Q.; Xie, F.; Tong, Y.; Habisch, R.; Yang, B.; Zhang, L.; Müller, R.; Fu, C. Dual-function chromogenic screening-based CRISPR/Cas9 genome editing system for actinomycetes. Appl. Microbiol. Biotechnol. 2020, 104, 225-239. [CrossRef]

49. Wang, K.; Zhao, Q.-W.; Liu, Y.-F.; Sun, C.-F.; Chen, X.; Burchmore, R.; Burgess, K.; Li, Y.-Q.; Mao, X.-M. Multi-layer controls of Cas9 activity coupled with ATP synthase over-expression for efficient genome editing in Streptomyces. Front. Bioeng. Biotechnol. 2019, 7, 304. [CrossRef]

50. Muth, G. The pSG5-based thermosensitive vector family for genome editing and gene expression in actinomycetes. Appl. Microbiol. Biotechnol. 2018, 102, 9067-9080. [CrossRef] 
51. Wlodek, A.; Kendrew, S.G.; Coates, N.J.; Hold, A.; Pogwizd, J.; Rudder, S.; Sheehan, L.S.; Higginbotham, S.J.; Stanley-Smith, A.E.; Warneck, T.; et al. Diversity oriented biosynthesis via accelerated evolution of modular gene clusters. Nat. Commun. 2017, 8. [CrossRef]

52. Najah, S.; Saulnier, C.; Pernodet, J.-L.; Bury-Moné, S. Design of a generic CRISPR-Cas9 approach using the same sgRNA to perform gene editing at distinct loci. BMC Biotechnol. 2019, 19, 18. [CrossRef] [PubMed]

53. Zetsche, B.; Heidenreich, M.; Mohanraju, P.; Fedorova, I.; Kneppers, J.; DeGennaro, E.M.; Winblad, N.; Choudhury, S.R.; Abudayyeh, O.O.; Gootenberg, J.S. Multiplex gene editing by CRISPR-Cpf1 using a single crRNA array. Nat. Biotechnol. 2017, 35, 31-34. [CrossRef] [PubMed]

54. Zheng, Y.; Su, T.; Qi, Q. Microbial CRISPRi and CRISPRa Systems for Metabolic Engineering. Biotechnol. Bioprocess. Eng. 2019, 1-13. [CrossRef]

55. Baltz, R.H. Streptomyces temperate bacteriophage integration systems for stable genetic engineering of actinomycetes (and other organisms). J. Ind. Microbiol. Biotechnol. 2012, 39, 661-672. [CrossRef]

56. Katayama, K.; Mitsunobu, H.; Nishida, K. Mammalian synthetic biology by CRISPRs engineering and applications. Curr. Opin. Chem. Biol. 2019, 52, 79-84. [CrossRef]

57. Rees, H.A.; Liu, D.R. Base editing: precision chemistry on the genome and transcriptome of living cells. Nat. Rev. Genet. 2018, 19, 770-788. [CrossRef]

58. Mishra, R.; Joshi, R.K.; Zhao, K. Base editing in crops: Current advances, limitations and future implications. Plant. Biotechnol. J. 2020, 18, 20-31. [CrossRef]

59. Arazoe, T.; Kondo, A.; Nishida, K. Targeted nucleotide editing technologies for microbial metabolic engineering. Biotechnol. J. 2018, 13, 1700596. [CrossRef]

60. Kim, J.-S. Precision genome engineering through adenine and cytosine base editing. Nat. Plants 2018, 4, 148-151. [CrossRef]

61. Komor, A.C.; Badran, A.H.; Liu, D.R. Editing the genome without double-stranded DNA breaks. ACS Chem. Biol. 2018, 13, 383-388. [CrossRef]

62. Molla, K.A.; Yang, Y. CRISPR/Cas-mediated base editing: Technical considerations and practical applications. Trends Biotechnol. 2019, 37, 1121-1142. [CrossRef]

63. Banno, S.; Nishida, K.; Arazoe, T.; Mitsunobu, H.; Kondo, A. Deaminase-mediated multiplex genome editing in Escherichia coli. Nat. Microbiol. 2018, 3, 423-429. [CrossRef] [PubMed]

64. Billon, P.; Bryant, E.E.; Joseph, S.A.; Nambiar, T.S.; Hayward, S.B.; Rothstein, R.; Ciccia, A. CRISPR-mediated base editing enables efficient disruption of eukaryotic genes through induction of STOP codons. Mol. Cell 2017, 67, 1068-1079. [CrossRef] [PubMed]

65. Xu, T.; Li, Y.; Shi, Z.; Hemme, C.L.; Li, Y.; Zhu, Y.; Van Nostrand, J.D.; He, Z.; Zhou, J. Efficient genome editing in Clostridium cellulolyticum via CRISPR-Cas9 nickase. Appl. Environ. Microbiol. 2015, 81, 4423-4431. [CrossRef] [PubMed]

66. Zhou, Y.; Lin, L.; Wang, H.; Zhang, Z.; Zhou, J.; Jiao, N. Development of a CRISPR/Cas9n-based tool for metabolic engineering of Pseudomonas putida for ferulic acid-to-polyhydroxyalkanoate bioconversion. Commun. Biol. 2020, 3, 1-13. [CrossRef] [PubMed]

67. Standage-Beier, K.; Zhang, Q.; Wang, X. Targeted large-scale deletion of bacterial genomes using CRISPR-nickases. ACS Synth. Biol. 2015, 4, 1217-1225. [CrossRef] [PubMed]

68. Song, X.; Huang, H.; Xiong, Z.; Ai, L.; Yang, S. CRISPR-Cas9 ${ }^{\text {D10A }}$ nickase-assisted genome editing in Lactobacillus casei. Appl. Environ. Microbiol. 2017, 83, e01259-17. [CrossRef]

69. Li, K.; Cai, D.; Wang, Z.; He, Z.; Chen, S. Development of an efficient genome editing tool in Bacillus licheniformis using CRISPR-Cas9 nickase. Appl. Environ. Microbiol. 2018, 84, e02608-e2617. [CrossRef]

70. Pyne, M.E.; Bruder, M.R.; Moo-Young, M.; Chung, D.A.; Chou, C.P. Harnessing heterologous and endogenous CRISPR-Cas machineries for efficient markerless genome editing in Clostridium. Sci. Rep. 2016, 6, 1-15. [CrossRef]

71. Chang, Y.; Su, T.; Qi, Q.; Liang, Q. Easy regulation of metabolic flux in Escherichia coli using an endogenous type I-E CRISPR-Cas system. Microb. Cell Fact. 2016, 15, 195. [CrossRef]

72. Vercoe, R.B.; Chang, J.T.; Dy, R.L.; Taylor, C.; Gristwood, T.; Clulow, J.S.; Richter, C.; Przybilski, R.; Pitman, A.R.; Fineran, P.C. Cytotoxic chromosomal targeting by CRISPR/Cas systems can reshape bacterial genomes and expel or remodel pathogenicity islands. PLoS Genet. 2013, 9, e1003454. [CrossRef] [PubMed] 
73. Cañez, C.; Selle, K.; Goh, Y.J.; Barrangou, R. Outcomes and characterization of chromosomal self-targeting by native CRISPR-Cas systems in Streptococcus thermophilus. FEMS Microbiol. Lett. 2019, 366, fnz105. [CrossRef] [PubMed]

74. Gong, T.; Tang, B.; Zhou, X.; Zeng, J.; Lu, M.; Guo, X.; Peng, X.; Lei, L.; Gong, B.; Li, Y. Genome editing in Streptococcus mutans through self-targeting CRISPR arrays. Mol. Oral Microbiol. 2018, 33, 440-449. [CrossRef] [PubMed]

75. Hidalgo-Cantabrana, C.; Goh, Y.J.; Pan, M.; Sanozky-Dawes, R.; Barrangou, R. Genome editing using the endogenous type I CRISPR-Cas system in Lactobacillus crispatus. Proc. Natl. Acad. Sci. USA 2019, 116, 15774-15783. [CrossRef] [PubMed]

76. Zhang, J.; Zong, W.; Hong, W.; Zhang, Z.-T.; Wang, Y. Exploiting endogenous CRISPR-Cas system for multiplex genome editing in Clostridium tyrobutyricum and engineer the strain for high-level butanol production. Metab. Eng. 2018, 47, 49-59. [CrossRef] [PubMed]

77. Zhang, J.; Li, X.; Deng, Z.; Ou, H.-Y. Comparative analysis of CRISPR loci found in Streptomyces genome sequences. Interdiscip. Sci. 2018, 10, 848-853. [CrossRef]

78. Qiu, Y.; Wang, S.; Chen, Z.; Guo, Y.; Song, Y. An active type I-E CRISPR-Cas system identified in Streptomyces avermitilis. PLoS ONE 2016, 11, e0149533. [CrossRef]

79. Biswas, A.; Gagnon, J.N.; Brouns, S.J.; Fineran, P.C.; Brown, C.M. CRISPRTarget: bioinformatic prediction and analysis of crRNA targets. RNA Biol. 2013, 10, 817-827. [CrossRef]

(C) 2020 by the authors. Licensee MDPI, Basel, Switzerland. This article is an open access article distributed under the terms and conditions of the Creative Commons Attribution (CC BY) license (http://creativecommons.org/licenses/by/4.0/). 